\title{
Los valores en las familias empresarias colombianas
}

Ernesto Barrera Duque

Recibido: Enero 2006

1 Profesor de tiempo completo del INALDE, Universidad de La Sabana en el área de Comercialización. Estudiante del Ph.D. en Administración de EAFIT. Master en Dirección de Empresas (MBA) por el IESE (España) -Universidad de Navarra-. Especialista en Economía Internacional y Abogado por la Universidad Externado. Actualmente se desempeña como consultor en el campo de la Empresa Familiar, la Estrategia y la Comercialización, y ha sido directivo durante varios años en el sector privado y público. 


\section{Introducción}

Este artículo tiene como objetivo identificar, con base en una muestra, los valores fundamentales en las familias empresarias colombianas, y establecer un modelo que permita su categorización. Los resultados de este estudio han sido el producto de un trabajo de campo con familias empresarias colombianas, y no de encuestas diligenciadas por un miembro determinado de la familia.

Los datos fueron obtenidos durante el acompañamiento y asesoría en procesos de planeación estratégica familiar (Ward y Carlock, 2003) a través de entrevistas personales y talleres grupales ${ }^{2}$ con la participación activa de la primera y la segunda generación ${ }^{3}$. El número de miembros familiares en cada uno de los procesos osciló entre seis y diez $z^{4}$.

Para efectos de este trabajo, la muestra comprende diez familias empresarias ubicadas en diferentes ciu- dades del país ${ }^{5}$. Las empresas vinculadas a las familias fueron clasificadas en una de las dos siguientes categorías: "empresas de trabajo familiar" $\mathrm{o}$ "empresas de dirección familiar" (Ga1lo, 1992). El período del estudio se refiere a entrevistas y talleres realizados durante los años 2004 y 2005. No se realizó ninguna segmentación por sectores ni por tamaño de las empresas familiares, dado que el interés se centró en la familia empresaria como tal.

\section{Un breve marco de referencia}

El protocolo familiar, antes que un negocio jurídico plasmado en un documento firmado por los miembros de la familia empresaria, es un proceso de diálogo familiar interpersonal propulsor de un pensamiento unificador en torno de un proyecto común. En este sentido, permite descubrir, reconocer, establecer y jerarquizar los valores declarados y en torno de los cuales

Dentro del marco de un proceso hacia el Protocolo Familiar.

3 Se trata de un estudio que define valores compartidos de la primera y segunda generación que coexiste además en la gestión empresarial.

4 Sin que para el efecto los miembros de la familia tuvieran conocimientos teóricos avanzados en el tema. Por tanto esta investigación no consiste en una elucubración teórica sobre los valores sino que pretende ser un reflejo de lo que ocurre en la realidad, en el seno de las familias empresarias.

5 La información sobre el nombre de las empresas y los valores que corresponden a cada familia empresaria son reservadas y, por tanto, no se mencionan en este artículo para mantener la confidencialidad.

6 Se incentiva a que un buen número de miembros de la familia trabajen en la empresa y su formación y preparación está orientada hacia este objetivo.

7 La familia tiene la intención de continuar con la propiedad de la empresa, pero se busca que sólo los más capacitados desde el punto de vista empresarial y directivo trabajen directamente en la empresa como miembros de junta directiva o en la alta gerencia. 
gira o debería girar la actividad empresarial de una familia empresaria.

Las personas (Pfeffer, 1998) y cultura de una organización (Gallo, 1992) son fuentes de ventajas competitivas. Así mismo, para una familia empresaria, sus miembros y su cultura generan una distinción específica de la manera como se piensa, actúa, se elige entre alternativas, se establecen responsabilidades, se establece una unidad de propósito, se toman decisiones y se resuelven conflictos. Los valores y la interpretación sobre la naturaleza humana también se reflejan en el estilo de liderazgo al interior de las empresas de la familia.

Jorge Yarce (2000) ha establecido que los valores son una ventaja competitiva y son la base sobre la cual se construye cualquier proceso de planeación estratégica familiar y empresarial. Son elementos esenciales de la cultura de una organización, y en este caso, de la dinámica de la familia empresaria, ya que condicionan el curso de sus decisiones, sus supuestos sobre la realidad, impactando en la dirección de las empresas ${ }^{8}$.
Los valores conforman el marco de referencia axiológico con los que los miembros de la familia priorizan sus preferencias, decisiones y acciones en relación con la familia misma y sus empresas, tanto en el largo como en el corto plazo.

De otro lado, también condicionan la interacción y operatividad práctica del modelo los tres círculos de la empresa familiar: familia, propiedad y empresa (Davis y Tagiuri, 1987).

Así mismo, no cabe duda de la importancia de los valores del fundador en la práctica y definición de los mismos (García Álvarez, 2001). Sin embargo, para efectos de este artículo se involucró activamente también a la segunda generación, dando paso más a la experiencia colectiva del grupo familiar.

En términos de causa-efecto, aquí se asume la hipótesis de que los valores de una familia empresaria son fuentes de ventaja competitiva fundamentales para la empresa familiar de su propiedad (Veciana et al, 1996) y de que existe el deseo de la familia de trasladarlos a la empresa.

8 Por ejemplo los valores establecen los criterios específicos para la asignación de recursos como en el caso de la preferencia hacia la reinversión de las utilidades para el crecimiento en lugar de la distribución de las mismas, bajo el valor de la austeridad personal. Estos valores también influyen en la manera como se otorga liquidez a los propietarios familiares, el riesgo empresarial que se asume y en nivel de endeudamiento. La justicia como valor influye en la manera como se realiza la sucesión patrimonial y los montos de los salarios y los modos de los flujos económicos para los miembros de la familia. Entre otros aspectos donde influyen los valores son la apertura para alianzas estratégicas, el ingreso de socios externos, el ingreso de directivos externos y el impacto en el sistema de selección, remuneración, evaluación y promoción de los directivos de las empresas. 


\section{Resultados del estudio}

Se tomaron los ocho valores más importantes de cada una de las familias empresarias analizadas, otorgándole a cada uno un puntaje de ocho a uno, de manera descendente, según su posición dentro de la jerarquía definida en cada caso.

De la combinación de los diez ca- sos mediante la sumatoria del puntaje atribuido a cada valor (frecuencia combinada con la jerarquía) resultó un puntaje total individual a través del cual se le asignó un puesto relativo.

De debe anotarse que se trata de los valores que son valiosos para la familia empresaria como bases del proyecto común iniciado a partir del proceso del Protocolo Familiar.

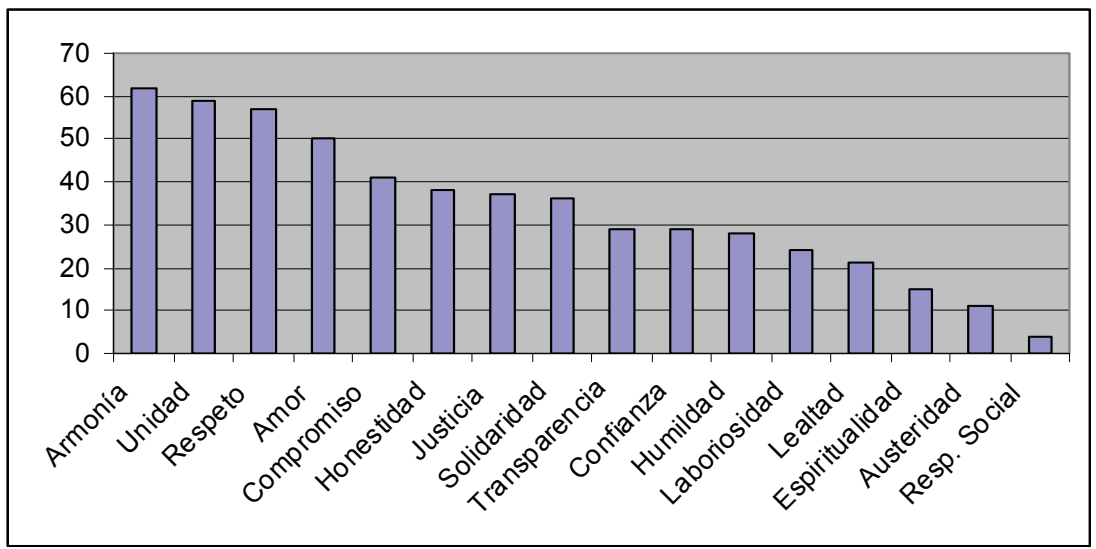

Tabla N. ${ }^{\circ}$ 1. Valores de las Familias Empresarias Colombianas, y su importancia relativa.

Como se observa en la tabla No. 1 , los principales valores de las familias empresarias colombianas son, según la muestra: la armonía, la unidad, el respeto, el amor y el compromiso.

Los dos primeros y el quinto coinciden con lo que algunos autores clásicos han determinado como las fortalezas de la empresa familiar (Gallo y Amat, 2003).

\section{Análisis del resultado}

\section{El contenido de los valores}

A continuación se presenta una síntesis sobre el contenido de los valores según lo que expresaron las familias empresarias, tanto en las entrevistas individuales como en los talleres realizados. A continuación se ordenan de acuerdo con la jerarquía encontrada en este estudio: 
Armonía (62): las relaciones entre los miembros de la familia se fundamentan en el respeto mutuo, la ponderación y el equilibrio dentro la diversidad. La interacción interpersonal se basa en la facultad de escuchar con empatía las posiciones y opiniones de los otros, entregando toda la información necesaria, sin que las tensiones emocionales afecten el buen desempeño de las reuniones familiares y las actividades empresariales y directivas.

Unidad (59): los miembros de la familia cooperan y se ayudan mutuamente para conseguir los objetivos e intereses del grupo, aprovechando las capacidades complementarias, trabajando en equipo, compartiendo los conocimientos en beneficio de todos $\mathrm{y}$ construyendo conjuntamente el futuro de la empresa familiar. Representa la voluntad de continuar cohesionados en el proyecto empresarial familiar.

Respeto (57): las relaciones entre los miembros de la familia se basan en el diálogo constructivo, en escuchar con tolerancia y comprensión, con cordura sin elevar el nivel de la voz. Todos los miembros de la familia se caracterizan por un comportamiento educado, de exaltación de la dignidad del otro y expresando cariño por los demás. Se acepta al otro como es, brindándole un trato amable y entendiendo las discrepancias para encontrar en ellas la riqueza de cada uno. Además, en la empresa se respetan las jerarquías formales.

Amor (50): en las relaciones familiares se busca el Bien de cada uno de sus miembros y existe una actitud de servicio desinteresado por las diferentes personas que componen la familia. Se propende el encuentro y la interacción personal y la disponibilidad para el otro. Existe una entrega y aceptación de los demás miembros familiares, con sus virtudes y defectos.

Compromiso (41): es una actitud de cumplir responsablemente las promesas y los acuerdos. En las actuaciones se imprime empeño buscando el mejor desempeño, obedeciendo siempre a los principios y valores familiares. El compromiso se materializa en la entrega y esfuerzo personales. Consiste en el trabajo hacia el mismo objetivo, con amor por los proyectos y por lo que se hace.

Honestidad (38): las actuaciones son consistentes con la rectitud, observando siempre en ellas las normas éticas y legales. No se permiten conductas incorrectas frente al manejo de los recursos de la empresa y de la familia; ni existirán actividades que pongan en duda o en riesgo el patrimonio y el nombre de la familia ante la comunidad. Existe una coherencia entre lo que se dice y se hace, sin engañar o defraudar a los demás. Las utilidades del negocio deben obtenerse siempre de manera ética.

Justicia (37): a cada uno lo que le corresponde según su situación específica, reconociendo los derechos del otro y sin abusar injustamente de la capacidad de influencia o del poder en las decisiones. Se materializa mediante mecanismos de participación de los 
miembros de la familia, aplicando un trato proporcional y equitativo a la situación de cada uno de sus miembros. Los beneficios económicos que se reciban de la empresa familiar serán fruto del esfuerzo personal, el trabajo en equipo y la propiedad.

Solidaridad (36): existe un espíritu de ayudar al otro y socorrerlo en momentos difíciles, tanto en lo económico como en lo emocional. Ser miembro de la familia trae derechos pero también obligaciones. Éstas son principalmente la preocupación activa por el Bien y bienestar de los demás miembros de la familia en sus diferentes dimensiones: psico-afectiva, intelectual, familiar, espiritual, material y social.

Transparencia (29): las intenciones de los actos serán visibles para todos los miembros de la familia. La información sobre la situación personal y profesional de cada miembro se informa con claridad y veracidad a todos los integrantes de la familia. La comunicación interpersonal se realiza con sinceridad y sin ambigüedades. No se desarrollan negocios que generen dudas en el seno de la familia. Las comunicaciones son fluidas, claras, veraces, confiables, oportunas, útiles, completas y motivadoras.

Confianza (29): es la credibilidad en la recta actuación de los demás. Se construye con base en la transparencia y bajo el compromiso de juzgar al otro rectamente, entendiendo que actúa siempre con base en sus buenas intenciones y por el Bien del grupo. Se trata de actuar con lealtad y con fun- damento en los criterios éticos. En la familia, la confianza implica considerar que los demás miembros hacen bien su trabajo, de manera honesta, y poniendo su mayor esfuerzo y recto criterio.

Humildad (28): es la actitud de reconocer que cada uno tiene debilidades y límites en sus capacidades y habilidades. Es aceptarse y aceptar a los demás por lo que son y no por lo que tienen. Actuar con sencillez y evitar imponer el propio punto de vista por el afán de dominar a los demás. Se trata de estar disponibles para servir a los demás. Las actuaciones están orientadas para lograr el Bien sin llamar la atención, ni requerir el aplauso ajeno para obrar correctamente. De esta actitud humilde se deriva un rechazo hacia la soberbia personal y cada uno propicia la espontaneidad y la apertura permanente al perdón. Es también consiste en reconocer con naturalidad los errores, aceptarlos y comprometerse con su solución y superación.

Laboriosidad (24): los miembros de la familia se caracterizan por su trabajo duro y arduo, realizado con persistencia y sacrificio, buscando la excelencia en lo que hacen. Mediante el propio comportamiento se dará ejemplo a los demás en términos del esfuerzo impregnado al trabajo. El éxito de la empresa familiar se entenderá como consecuencia de la calidad humana, el trabajo, el respeto por la dignidad de la persona humana, el liderazgo y las competencias profesionales. 
Lealtad (21): consiste en actuar con integridad y de acuerdo con los valores de la familia. Es un vínculo que une a la familia y se construye con base en la fidelidad a los compromisos y principios familiares, expulsando las traiciones, sin hablar mal del otro y respondiendo a las expectativas que se tienen de cada uno.

Espiritualidad (15): la existencia de un compromiso con la divinidad para aplicar y desarrollar los talentos recibidos. Es obedecer en las actuaciones a los principios, normas y virtudes exigidas por Dios, ofreciéndole un fuerte sentido de gratitud por pertenecer a la familia.

Austeridad (11): es vivir con sobriedad y sencillez, y consistente con las propias posibilidades económicas, ahorrando y haciendo un buen uso de los recursos disponibles, evitando los excesos materiales.

Responsabilidad social (4): existe un compromiso de reciprocidad con los empleados y la sociedad. A los empleados debe tratárseles con respeto, generando un buen ambiente de trabajo, motivándolos y promocionando siempre su potencial como personas y exaltando su dignidad humana. La familia debe retribuir a la comunidad en la cual desarrolló sus negocios principales. El espíritu emprendedor debe potenciarse para generar fuentes de empleo en la comunidad a la que se pertenece.

\section{La jerarquía de los valores}

El modelo ELISA de virtudes en los miembros de las familias empresarias postula que la exigencia, la laboriosidad, la iniciativa, la sencillez y la austeridad son las más importantes y fundamentales para garantizar una actividad empresarial longeva (Gallo y Vilaseca, 1996). En el caso colombiano esto es cierto, pero el orden jerárquico cambia un poco. Se han conformado tres grupos de valores según unos rangos iguales de veinte puntos cada uno: el primer grupo y etiquetado como el más importante va de 40 a 60 puntos, el segundo de 20 a 40, y el tercero de 0 a 20.

En el primer grupo denominado estructural (valores estructurales), se encuentran los valores más importantes y críticos para las familias empresarias: la armonía, la unidad, el respeto, el amor y el compromiso.

El esquema de los tres círculos ${ }^{9}$ (Davis y Tagiuri, 1987) de la empresa familiar tiene como fundamento y sustento la armonía en su interacción ${ }^{10}$ (Belausteguigoitia, 2003). Sin embargo, este valor debe buscarse y configurarse previamente en la dinámica interna entre los miembros de la familia empresaria. En este sentido, la madurez de las personas y su satisfacción por hacer parte de la familia contribuyen a potenciar este valor. En la muestra analizada, se reconoce como el pilar para la paz interna familiar $y$

\footnotetext{
9 Empresa, familia y propiedad.

10 Se refiere a la interacción entre los tres círculos.
} 
pilar para un adecuado clima organizacional en la empresa.

La unidad en la familia aparece como el segundo en importancia. El compromiso de las personas con la empresa y la familia, aparece en el quinto lugar. Este resultado coincide en parte con lo establecido por Gallo y Amat (2003) sobre la importancia operativa de estos dos valores ${ }^{11}$ para la continuidad, y como elementos sobre los cuales descansan algunos de los secretos de las empresas familiares centenarias.

El respeto y el amor aparecen como valores importantes en la dinámica de la familia, en el tercero y cuarto lugar respectivamente. En la práctica la confusión familia-empresa genera conflictos sistemáticos que, entre otros efectos, erosionan la manera como los miembros de la familia se dirigen la palabra y desarrollan el trato interpersonal. Las familias empresarias en un momento $\mathrm{u}$ otro han experimentado estos procesos traumáticos de irrespeto, y deciden tomar este valor - el respeto- como estructural para construir las relaciones entre sus miembros, garantizando en el compromiso común un trato decoroso entre ellos. El amor se entiende como la preocupación por el Bien ajeno, la capacidad de servicio y el sentimiento intenso que vincula a los miembros entre sí por hacer parte de una familia.

En otro nivel jerárquico, se encuentra el segundo grupo, denominado bajo el nombre de los valores operativos causales, basado en que con su práctica se potencia y construye el contenido de los estructurales. De acuerdo con los resultados de este estudio, son en su orden: honestidad, justicia, solidaridad, transparencia, confianza, humildad, laboriosidad y lealtad.

La honestidad de los miembros de la familia es importante, tanto a nivel interno como externo, pues hace parte importante de la reputación y mantenimiento de las redes de la familia con su entorno. Se busca vigilar de cerca el comportamiento de los familiares, para evitar poner en duda el nombre de la familia. De otro lado, con el segundo valor dentro de este grupo, su énfasis no es tanto la justicia como tal, sino la equidad, especialmente en los flujos económicos de la empresa hacia sus miembros, y en el trato del fundador hacia sus hijos y nietos. Se considera que la inequidad genera tensiones internas, desorden, desobediencia, odios indeseables al interior de la familia, es decir, fractura la unidad.

Como expresión de la solidaridad, los miembros de las familias se ayudan entre sí, especialmente en el aspecto económico, y muestran el deseo de establecer un fondo de liquidez y ahorro de la familia que sirva para financiar especialmente los estudios y la salud de sus miembros (solidaridad interna) y así mismo esperan la ayuda recíproca en casos de calamidad económica.

La transparencia se considera la fuente de la confianza. Cuando la actividad empresarial no se maneja con un

11 La unidad y el compromiso como fortalezas de la empresa familiar. 
flujo constante de información de la empresa hacia los miembros familiares propietarios, se generan tensiones en ocasiones desbordadas por la desconfianza; especialmente de aquellos quienes no se encuentran vinculados directamente a las actividades empresariales. Sin embargo, este tema funciona en oportunidades al revés de lo que ocurriría en una empresa no familiar. En esta última la confianza se construye en el día a día, con el tiempo. En la empresa familiar la confianza se da por supuesta, y la falta de transparencia es la principal causa de fracturas internas.

La humildad se entiende como la buena disposición ante los demás y para escuchar críticas constructivas del propio trabajo o del carácter. A pesar de la riqueza material en estas familias, los miembros de la segunda generación muestran una preocupación por no alejarse de la realidad colombiana, de sus injusticias, del drama de la pobreza y, especialmente del diálogo directo con las personas que trabajan en la empresa como un reconocimiento y una práctica de la igualdad en las relaciones entre seres humanos, considerando al otro como un fin en sí mismo y no como un simple instrumento productor de dinero, es decir, un sentido profundo para la promoción de la dignidad humana.

La laboriosidad ${ }^{12}$, es uno de los valores que los fundadores establecen como fundamentales para transmitir a sus sucesores (García Álvarez, 2001: 268) y es la herramienta para garantizar la continuidad y el relevo generacional adecuado.

Alain Chanlat (2005) afirma que las empresas familiares se adecuan al modo de ser mítico ${ }^{13}$ y por este motivo su valor fundamental, especialmente en los fundadores, es la lealtad, cuestión que se confirma en este estudio, pero no con la contundencia expuesta por este autor.

En el tercer grupo, encontramos los aquí denominados trascendentes, y de los cuales hacen parte la espiritualidad y la responsabilidad social. Como ya lo había anotado Borrero (1976) la religión católica tiene una marcada influencia sobre el comportamiento, actitudes y valores de los colombianos, incluidos los de las familias empresarias.

Este fenómeno se observó igualmente en las primeras décadas del siglo XX en la "industrialización" antioqueña, en donde fue marcada la influencia católica en los valores de los fundadores y sus descendientes. En este sentido no es ajeno a los procesos de definición axiológica el valor de la espiritualidad, entendida ésta como la creencia en Dios y el respeto a sus preceptos, y entendiendo la riqueza como un don de la divinidad fruto del trabajo arduo, y no como una derivación plegada a un origen "aristocrático".

12 Entendido como trabajo duro.

13 De acuerdo con los modos de ser organizativos desarrollados por Renée Bedard. Los modos de ser son: sistemático, pragmático, relacional y mítico. 
La responsabilidad social se mantiene como un deseo de revertir y trascender a la comunidad los beneficios que ella le ha entregado a la familia, y en la mayor parte de los casos se materializa mediante la filantropía, las obras de caridad, eventos con la población de bajos recursos, así como la constitución y subsidio de una fundación para ofrecer oportunidades a la población de menor poder adquisitivo.

En cuanto a la austeridad, se trata de un valor operativo causal, pero se encuentra por su bajo puntaje en el rango del tercer grupo ${ }^{14}$. Este valor se identificó con mayor intensidad en la primera generación, pero como este estudio incluyó la segunda, es probable que por este hecho no haya obtenido un puntaje mayor; máxime si lo que se encuentra en la práctica es un deseo de mayor liquidez para la segunda generación. De otro lado, este valor puede confundirse con la tacañería, y produce un efecto de no realzarlo con fuerza durante el proceso hacia el Protocolo Familiar.

Con este estudio, se confirma entonces el modelo ELISA (Gallo y Vilaseca, 1996). Pero aquí se ha realizado una jerarquización de acuerdo con los datos obtenidos durante los talleres, permitiéndonos configurar tres grupos de valores con niveles de importancia distinta y relativa: los estructurales, los operativos causales y los trascen- dentes. Se podría hablar en este sentido de un nuevo modelo ${ }^{15}$ más amplio: el TOCE.

Gallo y Amat (2003) en su libro "Los secretos de las empresas familiares centenarias" expresan lo siguiente:

La continuidad de la empresa familiar requiere gestionar constructiva y simultáneamente las diferentes exigencias que tienen tanto la empresa familiar como la familia empresaria, es decir, la familia que es propietaria de la empresa familiar. En especial, a medida que una empresa va pasando por varias generaciones se va produciendo una creciente fragmentación de la propiedad y se hace más compleja y difícil la continuidad... Además, dado que vivimos en un entorno cada vez más dinámico, complejo y hostil es fundamental que la familia construya un proyecto empresarial compartido por todas las personas que la integran. Para ello es imprescindible tener o desarrollar una filosofía y valores comunes, y una visión de futuro compartida, basada en la experiencia del pasado y la ilusión en el futuro (Gallo y Amat, 2003: 167) -negritas fuera del texto-.

La práctica ha demostrado que la definición y jerarquización de valores permite a las familias empresarias construir su actividad económica con base en un marco de referencia axiológicopautas para construir una filosofía central compartida (Collins y Porras, 1995)generando unos lineamientos para

\footnotetext{
14 Ha sido ubicado en el segundo grupo a pesar del bajo puntaje.

15 Para efectos de una mejor recordación se invierte el orden jerárquico del grupo y la $\mathrm{T}$ (trascendente) se pone primero, OC en segundo lugar como (operativos causales) y, en tercer lugar la $\mathrm{E}$ (estructural).
} 
tomar decisiones sobre la familia, la empresa y la propiedad, creando las bases para edificar la unidad y el compromiso a largo plazo (Ward y Carlock, 2003: 90) y permitiendo así una reducción de los traumas familiares del pasado. En este sentido, los valores son útiles además como factores de resiliencia ${ }^{16}$ (Cyrulnik, 2003) para la familia empresaria que, mediante el proceso orientado hacia el Protocolo Familiar busca -entre otros efectos y partir de una situación solidificada de "cero traumas"- darle un sentido compartido a su proyecto común: la empresa familiar.

\section{Bibliografía}

AMAT, Joan, coordinador (2004). La sucesión en la empresa familiar, Ed. Deusto, Barcelona.

BORRERO, Luis Delfín y Ocampo, Alfredo (1976). Empresarios colombianos, Ed. Ficitec, Bogotá D.C.

BELAUSTEGUIGOITIA, Imanol (2004). Empresas familiares, Ed. McGrawHill, México.

CHANLAT, Alain (2005). El rombo filosófico y los cuatro modos de ser. Conferencia dictada en la Universidad Eafit. Medellín.

COLLINS, James y PORRAS, Jerry (1995). Empresas que perduran, Ed. Norma, Bogotá.

CYRULNIK, Boris (2003). El murmullo de los fantasmas. Ed. Gedisa, Barcelona.

DÁVILA, Carlos, compilador (2003).
Empresas y empresarios en la historia de Colombia XIX-XX, Ed. Norma, Bogotá.

DAVIS, J y TAGURI, R (1987). Bivalent attitudes of the family firm. No publicado, Santa Bárbara California, Owner Manager Business Institute.

GALLO, Miguel Ángel (1992). La cultura en la empresa familiar, Ed. IESE publishing DGN-457, Barcelona.

GALLO, Miguel Ángel (1995). Empresa Familiar, Ed. Praxis, Barcelona.

GALLO, Miguel Ángel y Amat, Joan (2003). Los secretos de las empresas familiares centenarias, Ed. Deusto, Barcelona.

GARCÍA ÁLVAREZ, María Ercilla (2001). Los valores del fundador y su influencia en la empresa familiar en Galicia. Tesis Doctoral, Universidad Autónoma de Barcelona, España.

MORALES, Hollmann (1999). A puro pulso, Ed. Intermedio Editores, Bogotá.

PFEFFER, Jeffrey (1998). La ecuación humana, Ed. Gestión 2000, Barcelona.

VECIANA, J.M, García E, Bornheim, P.S. (1996). Founder-family-firm peformance paradigm. A holistic research programme for family-own business. Proceedings of the family business network $7^{\text {th }}$ world conference. Edimburgo, Escocia.

WARD, John y CARLOCK, Randel (2003). La planificación estratégica de la familia empresaria, Ed. Deusto, Bilbao.

YARCE, Jorge (2000). Los valores son una ventaja competitiva, Ed. Liderazgo, Bogotá.

16 Capacidad humana para superar traumas y heridas del pasado. Para lograrlo se requiere de la solidaridad y confianza de los otros. 


\title{
RESÚMENES Y ABSTRACTS
}

\section{LOS VALORES EN LAS FAMILIAS EMPRESARIAS COLOMBIANAS}

\author{
Ernesto Barrera Duque
}

\section{RESUMEN}

Este artículo, a partir de una investigación cualitativa y práctica, propone un modelo general de análisis para los valores en las dinámicas operativas de las familias empresarias colombianas. En el cuerpo del texto, se identifican los valores vigentes, estableciéndose su contenido y jerarquía, en función de los resultados del trabajo de campo.

PALABRAS CLAVE: Familia empresaria, empresa familiar, valores.

\begin{abstract}
This article, from a qualitative and practical research, proposes a general model of analysis for the dynamic values in operative the families of the Colombian industralists. In the body of the text, the effective values are identified, settling down their content and hierarchy, based on the results of the work of field.
\end{abstract}

KEY WORDS: Family entrepreneur, familiar company, values. 


\title{
ANÁLISIS DEL DISEÑO DE LOS CONTRATOS EN EL PROCESO DE SUBCONTRATACIÓN DE LAS ORGANIZACIONES PÚBLICAS, EL CASO DE EMCALI EICE
}

\author{
Henry Caicedo Asprilla
}

\begin{abstract}
RESUMEN
Éste documento utiliza las categorías de la economía institucional para evaluar el impacto que tiene sobre las organizaciones públicas el proceso de outsourcing o subcontratación. Aquí se plantea el interrogante de hasta dónde es posible alcanzar la eficiencia de una organización pública cuando el proceso de subcontratación está determinado por contratos intrínsecamente incompletos. A partir de la información de una muestra de 198 contratos que Emcali firmó con proveedores entre los años 2001 y 2004, se halló que 128 estaban mal diseñados y sólo 70 cumplían con el requisito del buen diseño económico; este hallazgo permite verificar la presunción de la teoría económica institucional que afirma que aunque los contratos estén diseñados conforme a la ley, estos presentan fallas en el diseño económico y por consiguiente la empresa experimenta costos de transacción. En este docu-
\end{abstract}

mento se evidencia que en el caso concreto de Emcali existen dos variables que clasifican los contratos entre bien y mal diseñados: el valor de la inversión y el índice de riesgo de ejecución del contrato; mientras que las variables duración del contrato, el riesgo legal, empresas en licitación, garantías y pólizas de aseguramiento, consideradas fundamentales en el diseño contractual de una transacción no cumplen con el propósito de corregir las anomalías en los contratos. En este trabajo se avanza hasta plantear que existe una conexión entre el mal diseño de los contratos y las dificultades financieras y administrativas de Emcali, que llevó a la empresa a la crisis más grande de su historia.

PALABRAS CLAVE: Organizaciones públicas, outsourcing, análisis de los contratos, economía institucional, árboles de decisión. 


\section{ABSTRACT}

This paper uses the categories of the institutional economy to evaluate the impact that has on the public organizations the process of outsourcing or subhiring. The principal question is: until where considers it is possible to reach the efficiency of a public organization when the subhiring process is determined by intrinsically incomplete contracts. From the information of a sample of 198 contracts that Emcali signed whit suppliers between years 2001 and 2004, one was that 128 badly were designed and only 70 fulfilled the requirement of the good economic design, this finding allows to verify the economic presumption of the institutional theory that it affirms that although the contracts are designed according to the law, these contracts present faults in the economic design and therefore the company experiences transaction costs. This document evidences that in the Emcali's case there are two indicator that classify contracts between good and badly designed: the value of the investment and the index of risk of execution of the contract, whereas the indicators duration of the contact, the legal risk, companies in licitation, guarantees and insurance policies, were considered fundamental in the contractual design of a transaction do not correct the anomalies of the contacts. In this paper one advances when a connection is established between the wrong design of contracts and the financial and administrative difficulties of Emcali, that led the company to the greatest crisis of its history.

KEY WORDS: Public organizations, outsourcing, analysis of contracts, institutional economy, decition trees. 


\title{
¿DESDE UNA INGENIERÍA DEL ALMA HACIA UN "MANAGEMENT" DEL ESPÍRITU? ACERCA DE LA NOCIÓN DE PSICOLOGÍA EN EL DISCURSO ADMINISTRATIVO Y ORGANIZACIONAL
}

\author{
Héctor Bermúdez
}

\section{RESUMEN}

Este ensayo busca un acercamiento a la genealogía de lo que se reconoce en la administración como las "Ciencias del Comportamiento" y una crítica a la relación entre el pensamiento administrativo y aquella corriente particular de la psicología que ha sido incorporada a éste: lo que comúnmente se conoce como la psicología industrial pero que bien podría nombrarse como el conductismo organizacional.

Cabe decirse también, que, por el trabajo de consultoría especializada en Gestión Humana del autor, y por la docencia tanto en cursos de MBA para ejecutivos, como en programas para especialistas en administración de personal, fue posible articular esa experiencia de campo, con lo inferido del análisis teórico de la revisión documental. Así entonces, este trabajo busca apoyar la línea de investigación en estudios sobre la Gestión Humana.

PALABRAS CLAVE: Administración, Conductismo Organizacional, Psicoanálisis, Psicología Industrial, Organizaciones.

\begin{abstract}
This test looks for an approach of the genealogy of which a critic is recognized in the administration like "Sciences of the Behaviour" on the other hand, the article explain the relation between the administrative thought and that particular current of the psychology that has been incorporated to this one. This challenge is commonly known like the industrial psychology but that could well name like the organizational conductism.

It is possible also to say itself, that, by the work of consultancy specialized in Human Management of the author, and by teaching as much in courses of MBA for executives, like in programs for specialists in personnel administration, it was possible to articulate that experience of field, with the inferred thing of the theoretical analysis of the documentary revision. Then, this work looks for to support the line of research in studies on the Human Management.
\end{abstract}

KEY WORDS: Administration, Organizational Conductism, Psychoanalysis, Industrial Psychology, Organizations. 


\title{
LOS DESAFÍOS DEL DISEÑO ORGANIZACIONAL E INSTITUCIONAL EN UN MEDIO AMBIENTE GLOBALIZADOR
}

\author{
José G. Vargas Hernández
}

\section{RESUMEN}

Este trabajo tiene por objetivo analizar los desafíos de diseño organizacional e institucional en un medio ambiente globalizador. Este análisis se inicia a partir de las consideraciones de los procesos de globalización económica en la emergencia de las nuevas asimetrías del poder y su impacto en los procesos de cambio organizacional, institucional, del Estado y las políticas públicas y el diseño intergubernamental. La información y el conocimiento son insumos de las comunidades de aprendizaje y para la formación de redes. Una característica de los arreglos institucionales y nuevas formas organizacionales es la creación del poder en los individuos (empowerment)

PALABRAS CLAVE: Asimetrías del poder, diseño institucional, diseño organizacional, globalización

\begin{abstract}
This work must by objective analyze the challenges of organizational and institutional design in global environment. This analysis begins from the considerations of the processes of economic globalization in the emergency of the new asymmetries of the power and its impact in the processes of organizational, institutional change, of the State and the public policies and the intergovernment design. The information and the knowledge are raw material of the learning communities for the formation of networks. A characteristic of the institutional adjustments and new organizationals forms is the creation of the power in the individuals (empowerment)
\end{abstract}

KEY WORDS: Asymmetries of the power, institutional design, organizational design, globalization. 


\title{
LA REFORMA DEL ESTADO EN COLOMBIA: ENTRE EL AJUSTE FISCAL Y FORTALECIMIENTO DE LA PARTICIPACIÓN CIUDADANA
}

\author{
Rosalía Correa Young
}

\begin{abstract}
RESUMEN
La reforma del Estado en Colombia y la descentralización, desde que se aprobó el Acto legislativo 01 de 1986, pasando por los acuerdos institucionales, plasmados en la Constitución de 1991 y referidos a esta materia, así como la normatividad actual, son analizados en este artículo a la luz de los enfoques teóricos que estimularon un nuevo modelo de Estado, a partir de la percepción respecto del agotamiento del Estado de bienestar, interventor y asistencial.

El artículo explica la dinámica en Colombia frente a dos paradigmas: uno proveniente de la sociología y la ciencia política que define la reforma desde una perspectiva policéntrica y uno proveniente de la economía neoclásica que ofrece una perspectiva mercadocéntrica. La primera en-
\end{abstract}

cuentra fortalezas para la democratización, la equidad, la superación de los desequilibrios regionales y la inclusión social. La segunda, el posicionamiento de las lógicas del mercado con la reducción, o desaparición de la intervención del Estado en aquellas funciones que los privados pueden resolver más eficientemente. Uno y otro colocan a la sociedad civil en un protagonismo activo, sea político, en su relación con el Estado; sea económico, en su relación con el mercado, pero ambos la conciben como actor de primera línea.

PALABRAS CLAVE: Reforma del Estado, Descentralización, Democratización, Paradigma policéntrico, Paradigma mercadocéntrico, Ajuste Fiscal, Participación, Privatización 


\section{ABSTRACTS}

The reform of the state in Colombia and the decentralization, since legislative Act 01 of 1986 was approved, happening through the agreements institutional, shaped in the Constitution of 1991 and referred this matter, as well as the present normatividad, are analyzed in this article to the light of the theoretical approaches that stimulated a new model of state, from the perception respect to the exhaustion of the welfare state, auditor and healthcare.

The article explains dynamics in Colombia as opposed to two paradigms: one originating of sociology and political science that the reform defines from a policentric perspective and one originating of the neoclassic economy that offers a market perspective. First it finds strengths for the democratization, the fairness, the overcoming of the regional imbalances and the social inclusion. Second, the positioning of the logics of the market with the reduction, or disappearance of the intervention of the State in those functions that the prevailed ones can solve more efficiently. Both places to the civil society in an active protagonism, is politician, in their relation with the State; be economic, in his relation with the market, but both conceive it like actor of forward edge.

KEY WORDS: The reform of the State, Decentralization, Democratization, policentric Paradigm, market Paradigm, Fiscal Adjustment, Participation, Privatization. 


\section{TEORÍA ECONÓMICA Y ESTADO DEL BIENESTAR. UNA APROXIMACIÓN}

Fernando Salazar Silva

\section{RESUMEN}

El siguiente artículo realiza un tránsito por algunas posturas dentro de las ciencias económicas para discutir el EB y presentar alternativas concretas al mismo. El propósito de Keynes en su afán de hacer legitimar la configuración y consolidación del Estado de Bienestar, se discutirá en el contexto de las particularidades de carácter teórico económico. Si bien el keynesianismo pudo concretar desde las ciencias económicas las bases para acercar la teoría a la realidad social, no entendemos por qué otras escuelas no han logrado constituirse en la base teórica del Estado de Bienestar actual.

PALABRAS ClAVE: Estado de Bienestar, Pacto keynesiano, Ciencia económica, Monetarismo, Expectativas racionales, teoría Neoclásica, Neokeynesianismo.

\begin{abstract}
The following article makes a transit by some positions within economic sciences to discuss the EB and to present concrete alternatives to he himself. Keynes's intention in its eagerness to make legitimize to the configuration and consolidation of the Welfare State, will be discussed in the context of the particularities of economic theoretical character. Although the keynesianism could make specific from economic sciences the bases to approach the theory the social reality, we do not understand so that other schools have not managed to constitute themselves in the theoretical base of the Welfare State present.

KEY WORDS:

Welfare State, Keynesian Accord, Economic Science, Monetarism, Rational Expectations, Theorie Neoclassic, Neo-Keynesianism.
\end{abstract}




\title{
LA RESPONSABILIDAD CIVIL AUTOMOVILÍSTICA Y EL ASEGURAMIENTO OBLIGATORIO Y VOLUNTARIO: UN ESTUDIO EN EL DERECHO COLOMBIANO Y ESPAÑOL
}

\author{
Raquel Ceballos Molano
}

\begin{abstract}
RESUMEN
La accidentalidad vial en Colombia es una de las primeras causas de daños corporales y materiales en la población circulante y peatonal, que obliga, al menos en teoría, al propietario o conductor, a responder civilmente de sus consecuencias. Así, este trabajo de tesis plantea el problema de la tipicidad como "actividad peligrosa" y la dispersión normativa derivada de la responsabilidad civil automovilística y los mecanismos de reparación a través del seguro voluntario y obligatorio del automóvil, consagrada en la legislación Civil, Penal, Comercial, Código Nacional de Tránsito, y en Decretos y Resoluciones administrativas, además de la doctrina científica y jurisprudencial, lo que evidencia que el legislativo colombiano no ha prestado atención, desde el punto de vista técnico y jurídico a las políticas de Estado para armonizar en un solo estatuto la responsabilidad derivada de los accidentes de tránsito y su aseguramiento, como si ocurre en el derecho comparado, el ordenamiento jurídico europeo y español.
\end{abstract}

PALABRAS CLAVE: Accidente de transito, responsabilidad civil, seguro obligatorio, soat, seguro automoviles, daño, indemnizacion, reparacion integral.

\section{ABSTRACT}

The road crash in Colombia is one of the first causes of body and materials damages in the circulating and peatonal population, that forces, at least in theory, to the proprietor or conductor, to respond civilly of its consequences. Thus, this thesis work creates the problem of the type like "dangerous activity" and the normative dispersion derived from the automobile civil responsibility and the mechanisms of repair through voluntary and obligatory insurance of the automobile, consecrated in the Civil, Penal, Commercial legislation, National Code of Transit, and in Decrees and administrative Resolutions, in addition to the scientific and jurisprudencial doctrine, which evidence that the legislative Colombian has not paid attention, from the technical and legal point of view to the state policies to harmonize in a single statute the responsibility derived from the traffic accidents and its securing, as if happens in the compared right, the European and Spanish legal ordering.

KEY WORDS: Road accident, civil responsibility, surely obligatory, soat, surely automobiles, damage, indemnification, integral repair. 


\title{
ENVEJECIMIENTO DEL PERSONAL
}

\author{
Sergio Castrillón
}

\section{RESUMEN}

¿De que manera la literatura en ciencias administrativas ha abordado el estudio del envejecimiento del personal? ¿Cuáles son las razones que han motivado este interés en los últimos años? Cuáles son las lógicas subyacentes? Reconociendo las tendencias demográficas mundiales que reflejan el envejecimiento de la población; este artículo procura responder a esta preguntas, a partir de una revisión exhaustiva de las principales publicaciones que se han ocupado del tema en el contexto internacional. Nuestro propósito es aumentar la visibilidad de la problemática y contribuir al debate sugiriendo acciones que revaloricen su dimensión humana.

PALABRAS CLAVE: Administración - Envejecimiento del Personal - Recursos Humanos - Fuerza Laboral - Jubilación - Retiro - Competencias y Desempeño - Motivación - Entrenamiento

\section{ABSTRACT}

How Literature in administrative sciences is boarded the study of the ageing of the personnel? Which are the reasons that have motivated east interest in the last years? Which are the underlying logics? Recognizing world-wide demographic tendencies that reflect the ageing of the population; this article tries to respond to this questions, from an exhaustive revision of the main publications that have taken care of the subject in the international context. Our intention is to increase the visibility of the problematic one and to contribute to the debate being suggested actions that revalue their human dimension.

KEY WORDS: Administration Ageing of the Personnel - Human Resources -Labor Force - Retirement - Competitions and Performance - Motivation - Training 


\title{
RENDICIÓN DE CUENTAS: ESTRATEGIA PARA EL FORTALECIMIENTO DE LA DEMOCRACIA A TRAVÉS DE LA LIMITACIÓN DEL PODER
}

\author{
Carlos Wladimir Gómez Cárdena
}

\begin{abstract}
RESUMEN
Este artículo contiene como apuesta epistemológica la construcción de un modelo genérico de Rendición de Cuentas, el cual puede llegar a ser implementado en las diferentes entidades de naturaleza estatal. A partir de una elaboración teórico - conceptual de la Rendición de Cuentas y de una observación práctica de los primeros ejercicios de rendición de cuentas llevados a cabo en Colombia, se exploran diversas potencialidades e instrumentos que pueden llegar a fortalecer las democracias modernas a través fundamentalmente, de la limitación de los ejercicios de poder. En las democracias modernas son muchos los mecanismos formales de limitación del poder consignados en el conocido sistema de frenos y contrapesos, pero son muy pocos los mecanismos reales. La rendición de cuentas como práctica democrática puede presentarse como uno de estos últimos, en la medida en que la sociedad organizada explore y explote tales potencialidades.
\end{abstract}

PALABRAS CLAVE: Políticas Públicas / Administración Pública / Rendición de Cuentas / Democracia / Limitación del Poder.

\section{ABSTRACT}

This article contains like comely epistemological the construction of a generic model of Accountability, which can arrive to be implemented in the different organizations of state nature. Starting from a theoretical - conceptual elaboration of the Accountability and of a practical observation of the first exercises of Accountability carried out in Colombia, diverse potentialities and instruments are explored that can end up strengthening the modern democracies fundamentally to inclination, of the limitation of the exercises of power. In the modern democracies there are many formal mechanisms of limitation of the power consigned in the well-known system of check and balances, but there are very few real mechanisms. Accountability as democratic practice can be presented like one of these last ones, in the measure in that the organized society explores and exploit such potentialities.

KEY WORDS: Public Policies / Public Administration / Accountability / Democracy / Limitation of the Power. 